Article

\title{
A Vibration Analysis Based on Wavelet Entropy Method of a Scroll Compressor
}

\section{Tao Liu * and Zaixin Wu}

School of Mechanical and Electronical Engineering, Lanzhou University of Technology, Lanzhou 730050, China; E-Mail: wu_zaixin@sina.com

* Author to whom correspondence should be addressed; E-Mail: liutao1971@lut.cn; Tel.: +86-931-2973860; Fax: +86-931-2757293.

Academic Editor: Raúl Alcaraz Martínez

Received: 30 May 2015 / Accepted: 10 October 2015 / Published: 19 October 2015

\begin{abstract}
Vibration-based condition monitoring and fault diagnosis is an effective approach to maintain the reliable operation of a scroll compressor. Unfortunately, the vibration signal from the scroll compressor always has characteristics of being non-linear and non-stationary, which makes vibration signal analysis and fault feature extraction very difficult. To extract the significant fault features, a vibration analysis method based on Wavelet entropy is proposed in this paper. Two forms of the wavelet entropy, namely the wavelet space feature spectrum entropy (WSFSE) and the wavelet energy spectrum entropy (WESE), are defined to depict instantaneous characteristics of the local variation of the vibration signal. Four types of mechanical faulty vibration signal, namely unbalanced rotor, malfunctioning scroll, loosened mechanical assembly, and loosened bearing, are analyzed by using the proposed approach. The experimental results show that feature components and energy distribution of each fault signal is accurately identified and revealed, which proves that the combined application of WSFSE and WESE approach is a successful scheme for the vibration analysis of scroll compressors.
\end{abstract}

Keywords: vibration analysis; fault feature extraction; wavelet entropy; feature component; scroll compressor 


\section{Introduction}

Scroll compressors have been becoming popular in refrigeration and air conditioning products because of their high efficiency, low noise, fewer components, low vibration, light weight, and small size compared with other types of compressors [1-5]. The malfunctions or faults because of the damages of their key components, such as fixed and orbiting scrolls, during the operating process of scroll compressors may cause abnormal operation and, if not detected early, can cause emergency shutdown. Therefore, efficient and accurate fault diagnosis technique is very important for guaranteeing reliability and performance of a scroll compressor. Over the past two decades, a large number of methods have been proposed for fault detection and diagnosis, and many attractive research results [6-14] have been obtained. In these methods, vibration-based spectrum analysis to the vibration signals of scroll compressors has been used as a regular technique in recent years [14-16] because of its capability of extracting fault feature from the signals. However, there still are some disadvantages in condition monitoring and fault diagnosis of scroll compressors. These deficiencies lead to some troublesome problems, one of which is that the diagnosis process was influenced by sampling point, sampling frequency, and sampling time. As a result, the diagnosis reliability is low but the diagnosis cost is relatively high. In this situation, the conventional analysis methods will not be suitable, because these methods are based on the assumption that the main vibration signals generated by machines are stationary and linear, while the signals acquired from scroll compressors are nonlinear and non-stationary random signals caused by multiple excitation sources. A general method [17] combined with entropy and grey incidence degree has been put forward to identify several types of faults occuring in scroll compressors, but the accurate identifying and revealing of feature components and energy distribution of each typical fault signal is still lacking.

As a novel feature extraction tool, wavelet entropy (WE) has attracted worldwide attention of experts and scholars with the ability to analyze transient features of non-stationary signals. It combines wavelet decomposition and entropy to estimate the degree of disorder of a signal with a high time-frequency resolution. Recently, WE has been used in engineering signal surveys such as electroencephalography (EEG) testing, climate processes, machinery vibration detection, and fault diagnosis and so on [18-22]. The results of these studies have manifested its better performance in analyzing the variability and complexity of signals compared with traditional methods. Remarkably, Chen and Li [23] introduced wavelet entropy to the feature extraction of transient signals in power systems, which makes it possible for some special power signals, non-faulty indirect lightning strikes, to be analyzed more extensively and deeply than ever. Chen et al. [24] presented an evaluation method of bearing factory quality based on wavelet packet entropy flow manifold learning and applied it to detect different types of defects on the bearing components. Some applications of WE in signal analysis and processing have shown its outstanding performance in distinguishing real signals and noise in a noisy series [25] and fault-detecting for different stable and unstable power swing conditions [26].

In view of the above-mentioned facts, an approach to the fault identification of scroll compressors by WE is presented in the paper to obtain more accurate state information about a scroll compressor in operation. Because the vibration signals from scroll compressors are not sensible to traditional time-domain and frequency-domain analysis, two forms of WE, namely wavelet space feature spectrum entropy (WSFSE) and wavelet energy spectrum entropy (WESE), are constructed to analyze vibration 
signals from time-frequency domain to realized fault feature extraction and discrimination. The excellent performance of the proposed method is verified through qualitatively analyzing four types of typical faulty signals acquired from an operating scroll compressor. Results from the experiment show that the presented approach is effective in accurately identifying and depicting four typical faults that occur in scroll compressors.

\section{Entropy of Vibration Signal}

\subsection{Information Extraction of Continuous Wavelet Transform}

The outcome of continuous wavelet transform of the original signal is a 2D matrix with time-scale vector series [27,28]. Assume the wavelet decomposition at scale $j(j=1,2, \cdots M)$ is $D_{j}$, a $M \times N$ matrix $D_{M \times N}$ can be constructed by the decomposing results. Apply singular value decomposition (SVD) to the matrix $D_{M \times N}$ and a singular spectrum $\left\{\delta_{i}\right\}=\left(\operatorname{diag}\left(\delta_{1}, \delta_{2}, \cdots \delta_{k}\right), 0\right)$ can be derived. Thus, WSFSE can be defined as

$$
S_{1}=-\sum_{j=1}^{M} p_{j} \log p_{j}
$$

where

$$
p_{j}=\delta_{j} / \sum_{j=1}^{k} \delta_{j}
$$

where $k$ is the number of nonzero singular value, $M$ is the maximum of scale number, $p_{j}$ represents the proportion of $j$-th singular value in a singular spectrum. WSFSE is capable of quantitatively distinguishing time-frequency signals by using SVD method in which the correlated wavelet space is mapped to a linearly independent feature space. According to SVD theory, the lower the frequency of the components, the less the number of singular value that does not equal zero. Therefore, the less complex the analyzed signal, the more concentration of energy and the smaller the value of WSFSE.

\subsection{Information Extraction of Discrete Wavelet Transform}

In this section, wavelet energy spectrum entropy (WESE) is evolved on the basis of Mallat decomposition algorithm. Assume a discrete time signal $x(n)$ is decomposed by discrete wavelet at scale $j(j=1,2, \cdots, M)$ at the moment of $k(k=1,2, \cdots, N)$, and the detail coefficient $D_{j}(k)$ and approximation coefficient $A_{j}(k)$ can be obtained. Thus, the original signal can be expressed by

$$
x(n)=D_{1}(n)+A_{1}(n)=D_{1}(n)+D_{2}(n)+A_{2}(n)=\sum_{j=1}^{M} D_{j}(n)+A_{M}(n)=\sum_{j=1}^{M+1} D_{j}(n)
$$

where $A_{M}(n)$ is replaced by $D_{M+1}(n)$ to unify symbols.

The time-frequency domain WESE is usually defined as

$$
S_{2}^{\prime}=-\sum_{j=1}^{M} p_{j} \log p_{j}
$$

where 


$$
\begin{gathered}
p_{j}=E_{j} / \sum_{j=1}^{M} E_{j} \\
E_{j}=\sum_{k=1}^{N}\left|D_{j}(k)\right|^{2}(j=1,2, \cdots, M)
\end{gathered}
$$

Traditional entropy analysis can denote the uncertainty of some condition occurring on the whole time span, but it fails to analyze the uneven change of energy distribution, moreover, the local nonlinear feature and state information of non-stationary signals are unable to be obtained. In the entropy analysis, the probability of each state is considered equivalent, this lead to underestimation or ignorance of contribution of shock state, which usually has large signal amplitude and small probability of occurrence, to the information entropy.

Therefore, to obtain more accurate state information, a sliding-window $C_{n}$ ( $w$ in width) is defined upon wavelet restructuring coefficients $D_{j}(k)$. Assume a short-term window $w \in N$, sliding coefficient $\delta \in N$, number of moves of the window $m \in N$. Then a sliding-window is defined as

$$
C_{n}(n, w, \delta)=\left\{d_{j}(k), k=1+m \delta, \cdots, w+m \delta\right\}
$$

where $m=1,2 \cdots, M, M=(N-w) / \delta$.

Afterwards, the energy sum of high-frequency parts of the testing signal within a sliding-window $C_{n}$ at $j$ scale can be computed at $k$ moment

$$
E_{j C_{n}}=\sum_{k=1+m \delta}^{w+m \delta}\left|D_{j}(k)\right|^{2}
$$

The total signal energy within the sliding window is defined as

$$
E_{C_{n}}=\left|A_{j}(k)\right|^{2}+\sum_{j=1}^{M} E_{j C_{n}}
$$

where $\left|A_{j}(k)\right|^{2}$ represents the energy sum of low-frequency parts of the testing signal within the sliding-window $C_{n}$ at the same scale and moment.

The improved WESE based on sliding-window signal interception thus can be defined as

$$
S_{2}=\sum_{m=1}^{M} S_{m}
$$

where

$$
\begin{gathered}
S_{m}=-\sum_{j=1}^{J+1} p_{j} \log p_{j} \\
p_{j}=\frac{E_{j C_{n}}}{E_{C_{n}}}
\end{gathered}
$$

The value of improved WESE changes with number of moves of the sliding window, which enables observation of signal features in different windows on a variety of scale spaces. 


\section{Experiment and Analysis}

\subsection{Experiment and Vibration Data}

Vibration experiments are performed on prototypes of frequency-converted scroll compressor. The parameters of the electric motor are listed in Table 1. An overall view of the testing point distribution on the compressor is shown in Figure 1, where 15 piezoelectric sensors are used to acquire the vibration data. Sensor $1 \#$ is located on the center of the compressor head. Sensor $5 \#$ is fixed at the position where the two scrolls engage. Sensors $6 \#$ is on the positon where the balance is assembled. Sensor $7 \#$ is located on the compressor shell where the mechanical assembly is mounted. Sensor $8 \#$ is on the position where roller bearings are set. Sensors $2 \#$ to $8 \#$ are symmetrically placed with Sensors $9 \#$ to $15 \#$. The rotation speed of motor ranges from $2000 \mathrm{rpm}$ to $4000 \mathrm{rpm}$. The sampling frequency is $2000 \mathrm{~Hz}$ and the number of data points for each signal sample is 1024 .

Table 1. Parameters of electric machine.

\begin{tabular}{cc}
\hline Line frequency $(\mathrm{Hz})$ & 60 \\
\hline Electric current $(\mathrm{A})$ & 10 \\
\hline Rotor slot & 34 \\
\hline Stator slot & 24 \\
\hline Rotational speed $(\mathrm{Rpm})$ & $1800-7200$ \\
\hline Slip ratio & 0.0417 \\
\hline Differential frequency $(\mathrm{Hz})$ & 2.5 \\
\hline Fundamental frequency $(\mathrm{Hz})$ & 1955 \\
\hline
\end{tabular}

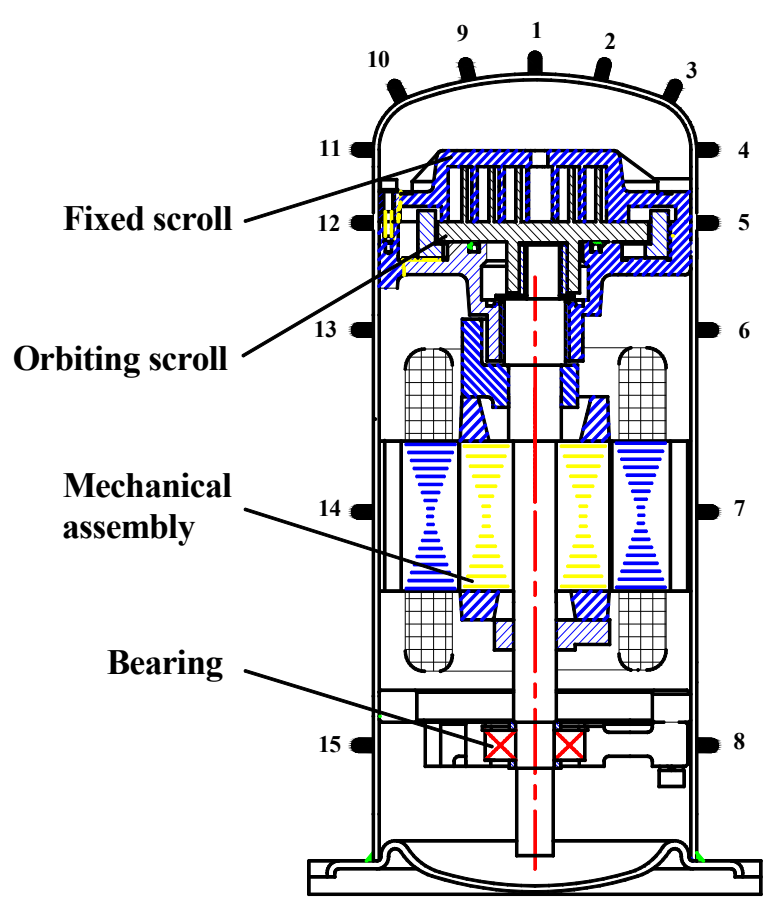

Figure 1. Overview of the sensor distribution on the scroll compressor. 
It was found that four types of mechanical faults, namely unbalanced rotor (Sensors 6\#), malfunctioning scroll (Sensor 5\#), loosened mechanical assembly (Sensor 7\#), and loosened bearing (Sensor 8\#), frequently occurred during the operation of the scroll compressor through lots of testing experiments and their analysis. The four types of faults under different speeds constitute the fault sample set and have been stored in the vibration test bench.

Figure 2 shows the raw vibration signals acquired from Sensors $5 \#$ to $8 \#$ located on positions where these four faults frequently occur. It can be easily seen that the time-domain vibration signals are heavily polluted by background noises. To capture and illustrate any fault characteristics is impossible through these time-domain wave-forms. To extract the fault features, the vibration signals need to be demodulated.

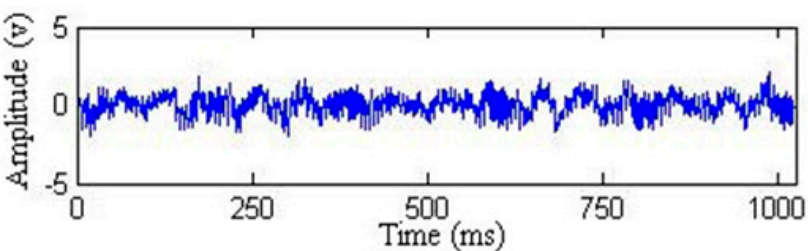

(a)

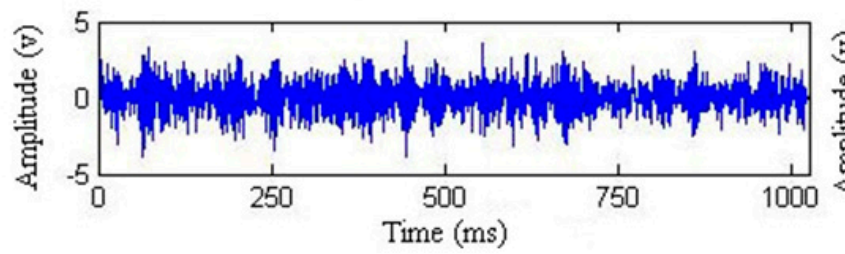

(c)

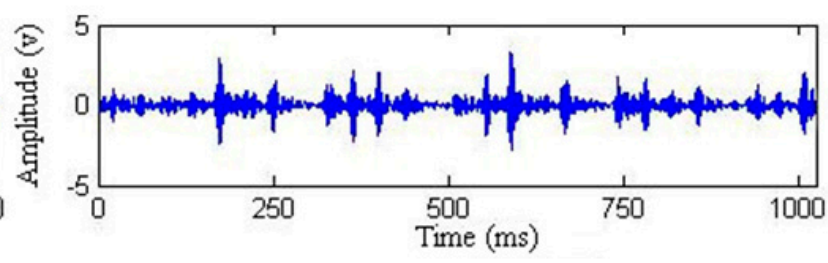

(b)

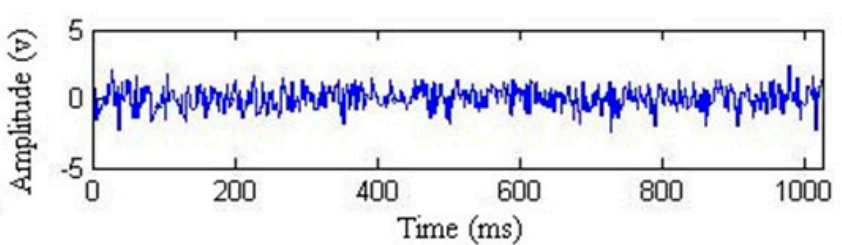

(d)

Figure 2. Waveforms of the vibration signals: (a) Sensor 6\#: unbalanced rotor; (b) Sensor 5\#: malfunctioned scroll; (c) Sensor 7\#: loosened mechanical assembly and (d) Sensor 8\#: loosened bearing.

\subsection{Analysis of Fault Vibration Signals}

To obtain useful information for vibration monitoring and analysis, the vibration signals from the four key positions on the compressor are processed by continuous wavelet transform at scale $j=64$, and their WSFSEs are illustrated in Figure 2, respectively.

As shown in Figure 3, a maximum value exists among the first 10-order singular values in the four different subgraphs, respectively, and the following entropy values tend to approach zero. In Figure 3a, two relatively large entropy values which represent dominant feature components just occur at the first two singular values. These feature components may be generated by dynamic eccentricity of the operating rotor. The component at the first-order singular value point has the greatest contribution to the entropy with a share of $63.25 \%$ of the total energy. Figure $3 \mathrm{~d}$ shows two larger WSFSE values, which account for $38.6 \%$ and $26.78 \%$ of the total energy, respectively, among the first seven-order singular values. This phenomenon confirms that such fault features as oil whip and dry friction arise when the energy of the loosened bearing is concentrated on multiple harmonic components $(2 \mathrm{X})$ and the fundamental harmonic component (1X). The above results show that the fault features can be effectively extracted from the vibration signal via WSFSE method. 


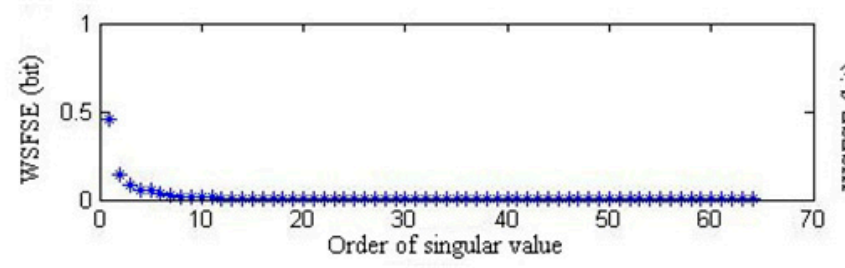

(a)

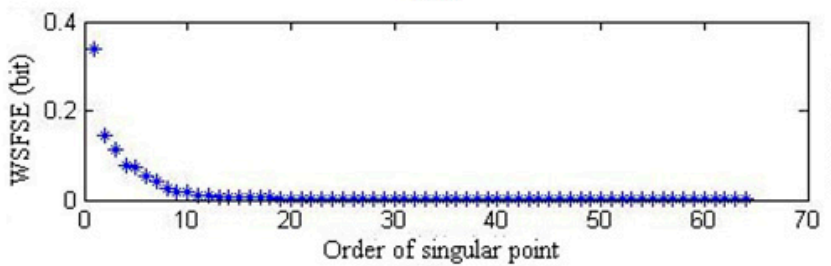

(c)

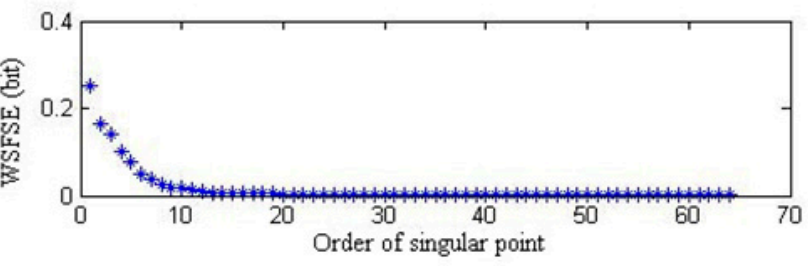

(b)

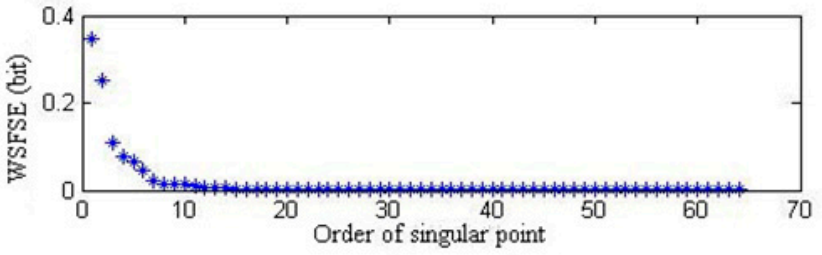

(d)

Figure 3. Wavelet feature spectrum entropy (WFSE) of the four faults: (a) unbalanced rotor; (b) malfunctioned scroll; (c) loosened mechanical assembly; and (d) loosened bearing.

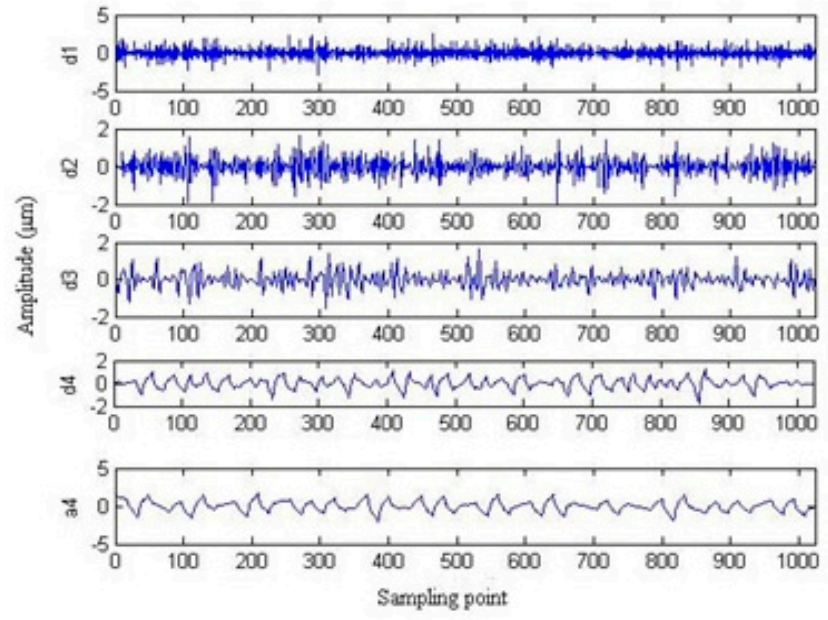

(a)

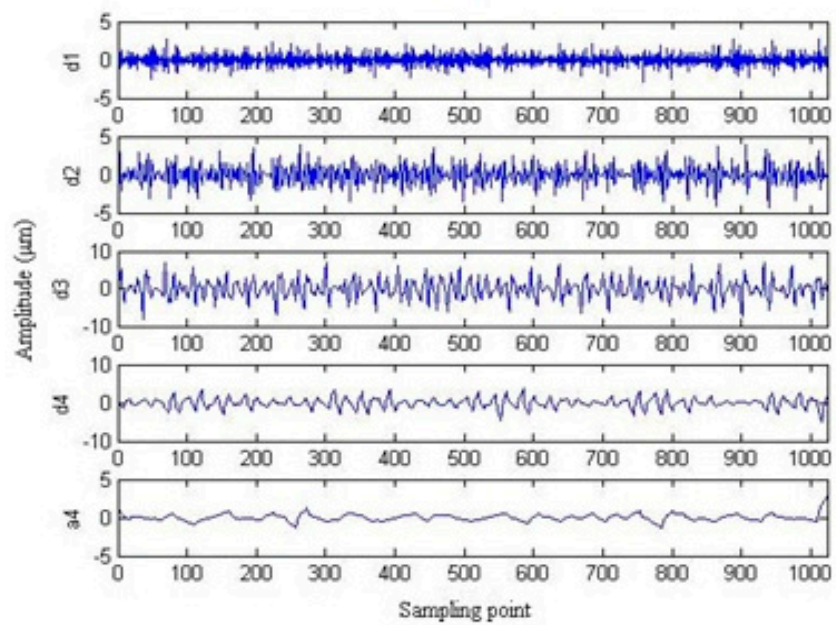

(c)

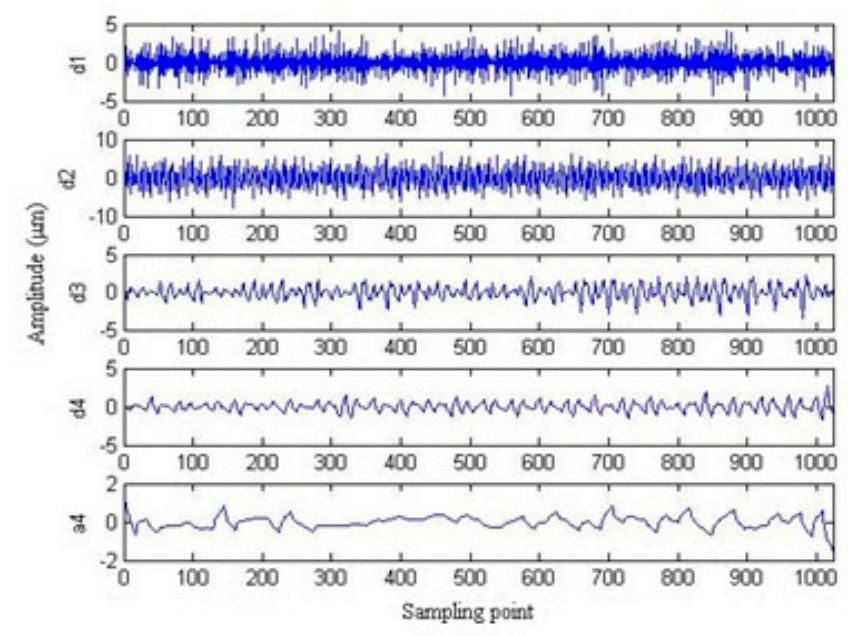

(b)

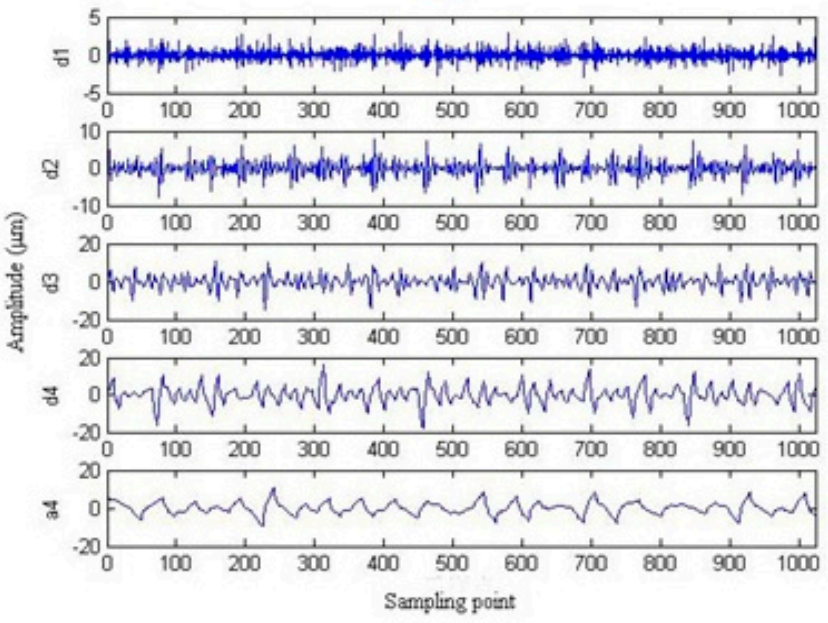

(d)

Figure 4. Reconstructed results of the vibration signal: (a) unbalanced rotor; (b) malfunctioned scroll; (c) loosened mechanical assembly; and (d) loosened bearing. 
To further acquire accurate global information and local features in multi-scale space, the four vibration signals are processed by multi-resolution one-dimension four-layer disperse wavelet decomposition on the basis of the $\mathrm{Db} 2$ wavelet because of its best smoothness for given support width. Each vibration signal is decomposed and reconstructed to form four signals $\mathrm{d} 1 \sim \mathrm{d} 4$ and an approximating signal a 4 to extract fault feature via WESE method. The reconstructed results of the vibration signals are illustrated in Figure 4, respectively.

The waveforms in Figure 4 are applied to a sliding window with window-length $w=100$ and step-length $\delta=10$. The WESE of the vibration signals are computed and depicted in Figure 5 .

It can be found that entropy value reaches a maximum at sampling point 100 and after that point, the entropy value remains relatively stable in Figure 5a. This result indicates that both single feature component and energy model occur when the rotor is not properly balanced. Special attention should be paid to Figure $5 b$ in which a visual feature representation of malfunctioned scroll is reflected. Before sampling point 189 , the entropy value remains approximately to 0.02 , which indicates that no obvious vibration happened at the fixed and orbiting scroll assembly during the stable operation of the compressor. At sampling point 190 and 300, the entropy value increases to 0.187 and 0.36 , respectively, because of the axial vibration of the orbiting scroll and the radial movement of the flexible mechanism in the assembly. Between sampling point 600 and 756, the entropy value changes from 0.05 to 0.29 . This occurrence may result from the uneven appearance of the nonlinear vibration caused by minor liquid slugging appearing in the scroll assembly, which leads to an increase of the uncertainty of vibration energy distribution and is reflected by entropy value. After that, the influence of liquid slugging on the compressor tends to gradually decrease and ends after sampling point 800 with stable entropy value.

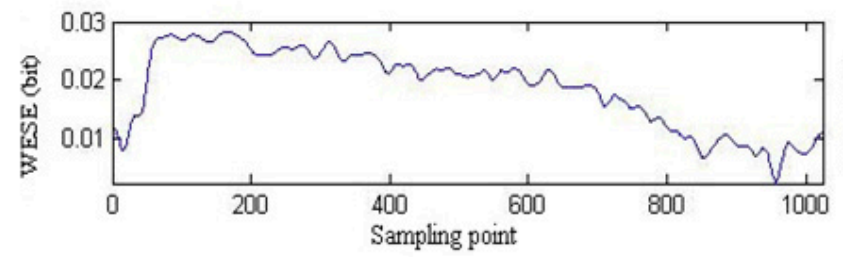

(a)

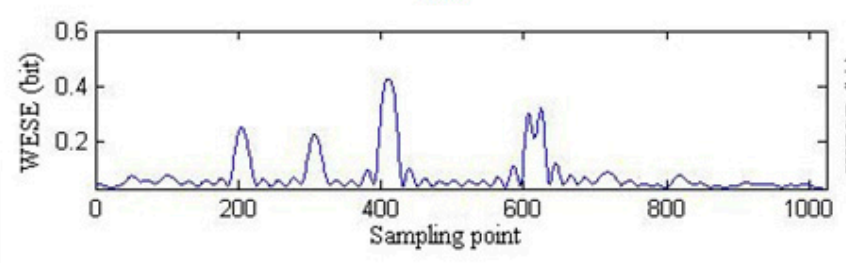

(c)

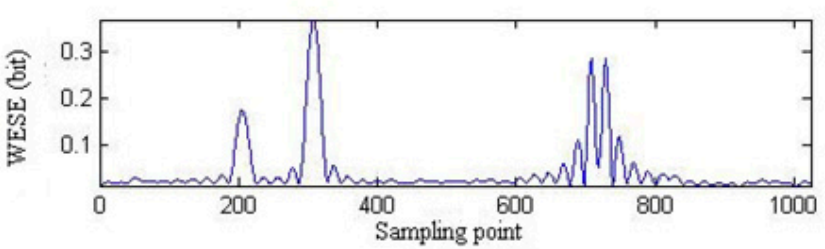

(b)

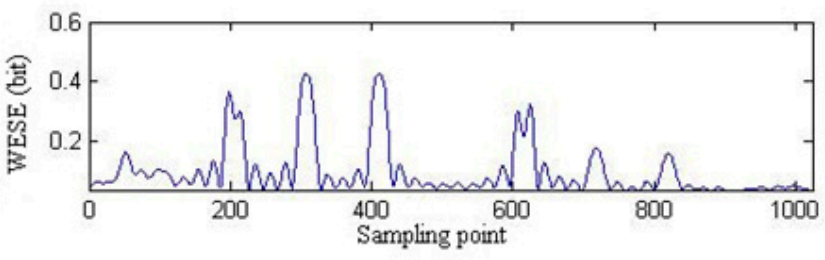

(d)

Figure 5. Wavelet energy spectrum entropy (WESE) of the four faults: (a) unbalanced rotor; (b) malfunctioned scroll; (c) loosened mechanical assembly and (d) loosened bearing.

Figure 5c shows that four feature components occur in the fault of loosened assembly. Comparatively, there are seven feature components with larger entropy value that occur in the fault of the loosened bearing, as shown in Figure 5d. This result reveals that there are more frequency components and their 
construction is more complicated with the fault of a loosened bearing than those in the fault of loosened assembly.

The above results demonstrate that the variation of the vibration pattern and the uncertainty of vibration energy distribution are sensitively detected using the WSFSE and WESE method. The reference samples of WSFSE and WESE for the four typical mechanical faults in the scroll compressor at the speed of $3400 \mathrm{rpm}$ are listed in Table 2.

The WSFSE and WESE methods are applied to extract fault features which are very difficult to be obtained by traditional frequency spectrum and power spectrum analysis. From Table 2, it is found that both WSFSE and WESE are relatively small in the fault of unbalanced rotor. Because when this kind of fault happens, the fundamental frequency based vibration is characterized by a single vibration pattern and concentrated energy, which leads to less uncertainty of the energy distribution. The cause of the fault may be the variation of the supporting force of the rotor generated by its uneven mass distribution and shifted center of mass.

Table 2. Wavelet entropy value of the fault sample at $3400 \mathrm{rpm}$.

\begin{tabular}{ccc}
\hline & WESE/Bit & WSFSE/Bit \\
\hline Unbalanced rotor & 1.2924 & 2.9594 \\
Malfunction scroll & 1.8749 & 4.0311 \\
Loosened mechanical assemby & 2.3637 & 4.1932 \\
Loosened bearing & 2.6864 & 4.8598 \\
\hline
\end{tabular}

For the fault of malfunctioned scrolls, relatively concentrated vibration energy appear in WSFSE and WESE analysis because liquid slugging within compressing chambers formed by both orbiting and fixed scrolls, as well as sympathetic vibration at their natural frequencies. The two combined causes result in unstable engagement of the scrolls and a larger vibration amplitude.

There are more frequency components and larger entropy values in the fault of loosened mechanical assembly. This may due to axial and radial frictions between rotating and fixed mechanical components within a loosened assembly during the long-term operation of the scroll compressor.

Likewise, In the fault of loosened bearing many vibration components, such as $1 / 2 X, 3 / 2 X, 1 X$, $\ldots, 4 X$, are generated because of excessive bearing wear or clearance, unsuitable bearing design, and varying parameters of lubricating oil. The resulted non-linear vibration appears non-uniformly in the time domain. Therefore, when this fault takes place, the complexity of the vibration energy distribution, which is reflected by the entropy value, is the largest among the four faults considered.

\section{Conclusions}

An approach to the fault identification of scroll compressors by WE is presented to overcome the shortcomings of the traditional spectrum analysis. Experimental study verifies that the proposed method can effectively extract significant fault features. The following conclusions are made as a result of the fault feature extraction of the scroll compressor using the combined approach of WFSE and WESE.

(1) Four types of typical mechanical faults during the operation of a frequency-converted scroll compressor are effectively identified. The feature components and energy distribution of each fault is accurately revealed. 
(2) Using the presented method provides reference samples and fundamental data for fault diagnosis of the scroll compressors. Furthermore, it will provide a solid stepping stone for a high quality design of scroll compressors, covering the structure design, key component optimization, manufacturing, and assembly aspects.

\section{Acknowledgments}

This study is supported by National Natural Science Foundation of China (Grant No. 51265027) and Fundamental Research Funds for the Universities in Gansu Province (Grant No. 1302ZTC034).

\section{Author Contributions}

Tao Liu contributed to the Section 2-4. Zaixin Wu mainly contributed to the Section 3. Both authors have read and approved the final manuscript.

\section{Conflicts of Interest}

The authors declare no conflict of interest.

\section{References}

1. Morishita, E.; Sugihara, M. Some design problems of scroll compressors. Bull. JSME 1986, 29, 4139-4146.

2. Cui, M.M. Numerical study of unsteady flows in a scroll compressor. J. Fluids Eng. Trans. ASME 2006, 128, 947-955.

3. Jiang, Z.; Harrison, D.K.; Cheng, K. Computer-aided design and manufacturing of scroll compressors. J. Mater. Process. Tech. 2003, 138, 145-151.

4. Winandy, E.O.; Saavedra, C.; Jean, L. Experimental analysis and simplified modeling of a hermetic scroll refrigeration compressor. Appl. Therm. Eng. 2002, 22, 107-120.

5. Dong, A.C.; Oh, K.K.; Myung, D.O. An experimental study on semiconductor process chiller using the digital scroll compressor. J. Mech. Sci. Technol. 2014, 28, 3345-3352.

6. Qu, L.S.; Xie, A.L.; Li, X. Study and performance evaluation of some nonlinear diagnostic methods for large rotating machinery. Mech. Mach. Theory 1993, 28, 699-713.

7. Samanta, B. Gear fault detection using artificial neural networks and support vector machines with genetic algorithms. Mech. Syst. Signal Process. 2004, 18, 625-644.

8. Kappaganthu, K.; Nataraj, C. Feature selection for fault detection in rolling element bearings using mutual information. J. Vib. Acoust. Trans. ASME 2011, 133, doi:10.1115/1.4003400.

9. Su, Z.; Tang, B.; Deng, L.; Liu, Z. Fault diagnosis method using supervised extended local tangent space alignment for dimension reduction. Measurement 2015, 62, 1-14.

10. Lee, Y.K.; Mavris, D.N.; Volovoi, V.V. A fault diagnosis method for industrial gas turbines using Bayesian data analysis. J. Eng. Gas Turbines Power 2010, 132, doi:10.1115/1.3204508.

11. Liu, H.N.; Liu, C.L.; Huang, Y.X. Adaptive feature extraction using sparse coding for machinery fault diagnosis. Mech. Syst. Signal Process. 2011, 25, 558-574. 
12. Wang, X.Y.; Makis, V. Autoregressive model-based gear shaft fault diagnosis using the Kolmogorov-Smirnov test. J. Sound Vib. 2009, 327, 413-423.

13. Xiang, L.; Yang, S.; Gan, C. Torsional vibration measurements on rotating shaft system using laser doppler vibrometer. Opt. Lasers Eng. 2012, 50, 1596-1601.

14. Yu, D.J.; Yang, Y.; Cheng, J.S. Application of time-frequency entropy method based on Hilbert-Huang transform to gear fault diagnosis. Measurement 2007, 40, 823-830.

15. Morsy, M.E.; Achtenová, G. Vehicle gearbox fault diagnosis based on cepstrum analysis. Int. J. Mech. Aerosp. Ind. Mechatron. Eng. 2014, 8, 1548-1554.

16. Cheng, J.S.; Yang, Y.; Yu, D.J. The envelope order spectrum based on generalized demodulation time-frequency analysis and its application to gear fault diagnosis. Mech. Syst. Signal Process. 2010, 24, 508-521.

17. Liu, T.; Wu, Z.X.; Wang, Y.W. Study on the fault diagnosis for scroll compressor based on information entropy. In Proceedings of the National Conference on Information Technology and Computer Science, Lanzhou, China, 16-18 November 2012; pp. 142-145.

18. Mahajan, R.; Morshed, B. Unsupervised eye blink artifact denoising of EEG data with modified multiscale sample entropy, kurtosis and wavelet-ICA. IEEE J. Biomed. Health Inform. 2015, 19, 158-165.

19. Pascoal, R.; Monteiro, A.M. Market efficiency, roughness and long memory in PSI20 index returns: Wavelet and entropy analysis. Entropy 2014, 16, 2768-2788.

20. Muralidharan, V.; Sugumaran, V. Feature extraction using wavelets and classification through decision tree algorithm for fault diagnosis of mono-block centrifugal pump. Measurement 2013, 46, 353-359.

21. Hu, Q.; He, Z.J.; Zhang, Z.S. Fault diagnosis of rotating machinery based on improved wavelet package transform and SVMs. Mech. Syst. Signal Process. 2007, 21, 688-705.

22. Bakhshi, A.D.; Bashir, S.; Loan, A. Application of continuous-time wavelet entropy for detection of cardiac repolarisation alternans. Signal Process. 2013, 7, 783-790.

23. Chen, J.; Li, G. Tsallis Wavelet entropy and its application in power signal analysis. Entropy 2014, 16, 3009-3025.

24. Chen, X.; Liu, D.; Xu, G.; Jiang, K. Application of wavelet packet entropy flow manifold learning in bearing factory inspection using the ultrasonic technique. Sensors 2015, 15, 341-351.

25. Sang, Y.F.; Wang, D., Wu, J.C. Entropy-based method of choosing the decomposition level in wavelet threshold de-noising. Entropy 2010, 12, 1499-1513.

26. Dubey, R.; Samantaray, S.R. Wavelet singular entropy-based symmetrical fault-detection and out-of-step protection during power swing. IET Gener. Transm. Dis. 2013, 7, 1123-1134.

27. Sang, Y.F.; Wang, Z.G.; Li, Z.L. Discrete wavelet entropy aided detection of abrupt change: A case study in the Haihe river basin, China. Entropy 2012, 14, 1274-1284.

28. Chou, C.M. Wavelet-based multi-scale entropy analysis of complex rainfall time series. Entropy 2011, 13, 241-253.

(C) 2015 by the authors; licensee MDPI, Basel, Switzerland. This article is an open access article distributed under the terms and conditions of the Creative Commons Attribution license (http://creativecommons.org/licenses/by/4.0/). 